\title{
Hipertensão arterial e fatores relacionados em adultos residentes em um território urbano precário do Nordeste Brasileiro
}

\author{
Hypertension and associated factors in adults in a low income urban area of Northeastern \\ Brazil
}
Hipertensión arterial y factores asociados en adultos en una zona urbana desfavorecida del noreste de Brasil

Lisianny Camilla Cocri do Nascimento Ferreira ${ }^{1 *}$, Silvia Pereira da Silva de Carvalho Melo², Pedro Israel Cabral de Lira ${ }^{1}$, Anete Rissin ${ }^{3}$, Malaquias Batista Filho ${ }^{3}$.

\begin{abstract}
RESUMO
Objetivo: Analisar a prevalência e os fatores relacionados à hipertensão arterial em adultos residentes em um território urbano precário, em Recife (PE). Métodos: Trata-se de um estudo transversal, com uma amostra de 663 adultos de 20 a 59 anos. Aqueles que apresentaram PA sistólica $\geq 140 \mathrm{mmHg}$ e/ou PA diastólica $\geq 90$ $\mathrm{mmHg}$ e/ou sob utilização de medicamento anti-hipertensivo, foram considerados com HAS. Analisaram-se possíveis associações da HAS com variáveis socioeconômicas, ambientais, biológicas, comportamentais, antropométricas e bioquímicas (morbidade), através de Regressão de Poisson, considerando-se como estatisticamente significantes aqueles com valor de $p<0,05$. Resultados: A prevalência de HAS encontrada foi de $36,9 \%$, sendo maior na faixa etária entre 50 e 59 anos, com aumento progressivo a partir dos 30 anos. Os fatores associados à HAS após os ajustes foram: faixa etária $(p<0,001)$, IMC $(p<0,001)$ e triglicerídeos $(p<0,001)$. Conclusão: A elevada prevalência de HAS e os fatores associados encontrados, impõe a necessidade de incluir este problema como prioridade de saúde pública nas comunidades carentes, visando sua prevenção e controle.
\end{abstract}

Palavras-chave: Baixa renda, Hipertensão, Pressão arterial, Prevalência.

\begin{abstract}
Objective: Analyse the prevalence of hypertension and the related factors in adults living in a low income area, in Recife (PE). Methods: A cross sectional study was conducted with a sample composed by 663 adults, with ages between 20-59 years old. High blood pressure was defined as systolic blood pressure $\geq 140 \mathrm{mmHg}$ and/or diastolic blood pressure $\geq 90 \mathrm{mmHg}$, and/or under drug treatment for blood pressure control. Possible associations of blood pressure with socioeconomic, environmental, biological, behavioral, anthropometric and biochemical variables (morbidity) were analyzed through Poisson Regression, considering as statistically significant those with $p$ value $<0.05$. Results: The prevalence of hypertension was $36.95 \%$, being greater in 50-59 years age group with progressive increase from 30's years old. Factors associated with high blood pressure after adjustment were: age-group $(p<0,001), \mathrm{BMI}(p<0,001)$ and triglycerides $(p<0,001)$. Conclusion: The hypertension prevalence and the associated factors found, imposes the need to include this problem as a priority of public health in the poor communities, aiming at its prevention and control.
\end{abstract}

Keywords: Low income, Hypertension, Blood pressure, Prevalence.

\footnotetext{
1 Universidade Federal de Pernambuco (UFPE), Recife - PE. *E-mail: lisiannyferreira@outlook.com

2 Instituto Aggeu Magalhães, Fundação Oswaldo Cruz (Fiocruz), Recife - PE.

${ }^{3}$ Instituto de Medicina Integral Prof. Fernando Figueira, Recife - PE.
}

PUBLICADO EM: 4/2021 


\section{RESUMEN}

Objetivo: Analizar la prevalencia y los factores asociados a la hipertensión arterial sistémica en adultos residentes en una zona urbana necessitada, en Recife (PE). Métodos: Se trata de un estudio transversal, con una muestra de 663 adultos de 20 a 59 años. Aquellos con PA sistólica $\geq 140 \mathrm{mmHg}$ y / o PA diastólica $\geq 90$ $\mathrm{mmHg}$ y / o bajo el uso de medicación antihipertensiva, se consideró que tenían HAS. Se analizaron las posibles asociaciones de HSA con variables socioeconómicas, ambientales, biológicas, conductuales, antropométricas y bioquímicas (morbilidad) mediante Regresión de Poisson, considerando como estadísticamente significativas aquellas con una $p<0.05$. Resultados: La prevalencia de HSA encontrada fue del 36,9\%, siendo mayor en el grupo de edad entre 50 y 59 años, con un aumento progresivo a partir de los 30 años. Los factores asociados a la hipertensión después de los ajustes fueron: rango de edad $(p<0,001)$, IMC $(p<0,001)$ y triglicéridos $(p<0,001)$. Conclusión: La alta prevalencia de hipertensión y los factores asociados encontrados, impone la necesidad de incluir este problema como una prioridad de salud pública en las comunidades necesitadas, con miras a su prevención y control.

Palabras clave: Bajos ingresos, Hipertensión, Presión arterial, Prevalencia.

\section{INTRODUÇÃO}

A hipertensão arterial tem alta prevalência no Brasil e no mundo, sendo considerada um dos maiores problemas de saúde pública e um importante fator de risco modificável para Doenças Cardiovasculares (CDV) (MALACHIAS MVB, et al., 2016; OLSEN MH, et al., 2016). Entre suas causas, evidencia-se na literatura a associação com a ingestão excessiva de sal, o consumo abusivo de álcool, a inatividade física, o excesso de peso, o tabagismo e os distúrbios do metabolismo da glicose e dos lipídios, além de fatores biológicos como genética e idade (SILVA EC, et al., 2016; MALTA DC, et al., 2017; ZANGIROLANI LTO, et al., 2018).

A prevalência mundial da hipertensão arterial sistêmica (HAS) é de aproximadamente $31 \%$, estima-se que em 2025 chegará a uma prevalência de 40\% (WHO, 2015; MOREIRA JPL, et al., 2013). No caso particular do Brasil, dados de 2017 do Sistema de Vigilância de Fatores de Risco e Proteção para Doenças Crônicas por Inquérito Telefônico (VIGITEL) mostraram que a frequência de diagnóstico médico prévio de hipertensão arterial foi de $24,3 \%$, sendo maior em mulheres $(26,4 \%)$ do que em homens $(21,7 \%)$ (BRASIL, 2018).

No novo cenário epidemiológico, observa-se no Brasil a tendência de aumento da prevalência das doenças crônicas não transmissíveis - DCNTs como uma das características marcantes do processo de transição nutricional e epidemiológica do país (MARINHO F, et al., 2016). Estudos em todo mundo tem evidenciando as desigualdades nos perfis de adoecimento, demonstrando que o risco de apresentar doenças do aparelho circulatório é maior nos grupos menos favorecidos.

Assim dentre as doenças que acometem populações em situações de vulnerabilidade social, a hipertensão arterial se apresenta com elevadas prevalências e fortemente associada a estratos sociais mais baixos, colocando o agravo como um grave problema no campo da saúde pública na atualidade, desde que suas consequências biológicas e sociais se tornam mais complexas (ARAÚJO ASS, et al., 2015; BEZERRA VM, et al., 2013; BEZERRA VM, et al., 2017; CHOR D, et al., 2015; VEENSTRA G, 2013; WHO, 2015; CHOR D, et al., 2015; ALMEIDA ND, 2013).

As mudanças comportamentais do século $\mathrm{XX}$, tais como hábitos de vida sedentários, mudanças nos padrões tradicionais de consumo alimentar (com destaque para a alta ingestão de sal), ingestão de álcool, características socioambientais sob constantes mudanças, susceptibilidade biológica, incluindo fatores genéticos, têm se destacado como condicionantes de risco ligados à hipertensão (BRASIL, 2011; MALACHIAS MVB, et al., 2016).

Por sua consistência, com os achados clínicos e epidemiológicos, os fatores sociodemográficos (sexo, idade, escolaridade, renda, raça/cor), vêm ganhando destaque embora diferenças na prevalência de HAS ou na especificidade destas associações mereçam ainda maiores investigações (SÁ NNB e MOURA EC, 2011). 
Diante da conjuntura sociodemográfica, ambiental e econômica ao qual a hipertensão arterial está inserida e o seu impacto no processo saúde/doença da população, principalmente situadas em comunidades urbanas carentes, justifica-se a realização deste estudo, que teve como objetivo, analisar a prevalência e os fatores relacionados à hipertensão arterial, em adultos entre 20 a 59 anos, residentes em um território urbano precário, em Recife, Nordeste do Brasil.

\section{MÉTODOS}

O presente trabalho é de corte transversal, e utilizou dados secundários a pesquisa maior intitulada "Saúde, nutrição e serviços assistenciais numa população carente do Recife: um estudo baseline", o qual foi realizado através de uma parceria entre as instituições: 1) Instituto de Medicina Integral Professor Fernando Figueira (IMIP); 2) Departamento de Nutrição da Universidade Federal de Pernambuco - DN/UFPE; e 3) Secretaria de Saúde do município do Recife. Entre os meses de junho a dezembro de 2014 ocorreu a coleta de dados, a qual teve base domiciliar, em uma região urbana carente, localizado no município de Recife, capital do Estado de Pernambuco.

Para esse estudo, tomou-se como base para o universo amostral os dados do Sistema de Informação de Atenção Básica (SIAB), o qual referia 3.816 adultos cadastrados, com idade compreendida entre 20 e 59 anos, em Recife no ano de 2013. Considerou-se uma prevalência de 32,7\% de HAS nos adultos nessa faixa etária, na Região Metropolitana do Recife de Pernambuco, segundo a III Pesquisa de Saúde e Nutrição (UFPE, 2012), realizada em Pernambuco. Assumiu-se um erro de estimativa de 3,5\% e um nível de confiança de $95 \%$, obtendo-se assim, uma amostra mínima inicial de 584 participantes. Sobre este valor foi aplicado um acréscimo de $10 \%$ na amostra, para compensar possíveis perdas ou problemas de não resposta, obtendo-se assim, uma amostra mínima de 643 participantes.

Para a seleção da amostra, do tipo probabilística os participantes foram selecionados a partir de uma relação de adultos residentes nos domicílios, através de sorteio aleatório simples, numa relação de um em cada quatro pessoas, sem substituição. Os critérios de exclusão para a pesquisa primária, e consequentemente para este trabalho, foram as pessoas que apresentavam braços com edema ou inadequados para as braçadeiras disponíveis (por folgas excessivas ao serem posicionadas no braço ou por não se manterem fixadas pelo velcro). O programa estatístico para cálculo amostral foi o Epi info versão 6.04 para estudos transversais.

A variável desfecho desse trabalho foi a hipertensão arterial e para sua medição foram utilizados procedimentos padronizados, realizados em dois momentos distintos. Considerou-se como casos de HAS os adultos que apresentaram pressão arterial sistólica $\geq 140 \mathrm{mmHg}$ e/ou de pressão arterial diastólica $\geq 90 \mathrm{mmHg}$ ou que relataram o uso de medicação anti-hipertensiva.

As variáveis independentes foram agrupadas em socioeconômicas, ambientais, comportamentais, antropométricas e de morbidade. As socioeconômicas avaliadas forma: a classe econômica (B1/B2/C1, C2 e $\mathrm{D} / \mathrm{E})$, a qual foi avaliada com base no critério da Associação Brasileira de Empresas de Pesquisa - ABEP (2014); escolaridade (analfabeto/fundamental 1 incompleto, fundamental 1 completo/fundamental 2 incompleto, fundamental 2 completo/médio incompleto e médio completo/superior incompleto/superior completo) e ocupação (trabalha, não trabalha e aposentado/pensionista/beneficiário); Já para as ambientais foram analisados o abastecimento de água (rede geral e outros), destino do lixo (coletado, terreno baldio e outros) e esgotamento sanitário (rede geral, curso de água e outros);

Quanto as variáveis biológicas, foi avaliado o sexo (masculino e feminino), faixa etária (20-29, 30-39, 4049 e 50-59) e cor autorreferida (branca, parda e preta) dos participantes. Em relação aos dados comportamentais, analisou-se o consumo de sal (adequado e inadequado), investigado e classificado com base no questionário de consumo alimentar semanal utilizado pelo Ministério da Saúde; tabagismo (não fuma, ex fumante e fumante) e atividade física (muito ativo, ativo e insuficientemente ativo) (BRASIL, 2014). Esta variável teve como referência o "Questionário Internacional de Atividade Física - IPAQ" versão curta, o qual classifica o nível de atividade física e, quatro categorias: muito ativo, ativo, irregularmente ativo e sedentário. Para fins de análise, as categorias irregularmente ativo e sedentário foram recategorizadas em insuficientemente ativo. 
Foram considerados ativos os indivíduos que referiram praticar atividade vigorosa com frequência maior ou igual a três vezes na semana por 20 minutos ou mais; ou indivíduos que praticavam atividade moderada ou de caminhada com frequência maior ou igual a cinco vezes na semana por 30 minutos no mínimo; ou ainda qualquer atividade que somada fosse equivalente a uma frequência maior ou igual a cinco vezes na semana e maior ou igual a 150 minutos na semana (caminhada + moderada + vigorosa). Todos os outros casos que não atenderam a esse critério foram considerados insuficientemente ativos (CRAIG CL, et al., 2003).

Para os dados antropométricas, a medida do peso foi realizada através de uma balança (SECA 876), com capacidade de até $250 \mathrm{Kg}$ e escala de 100 gramas. Os indivíduos foram pesados descalços, com indumentária mínima e sem qualquer objeto nos bolsos, nas mãos ou na cabeça. A altura foi determinada pelo estadiômetro portátil, da marca Alturaexata, Ltda, milimetrado, com precisão de até $(1 \mathrm{~mm})$ em toda a sua extensão. Tanto o peso quanto à altura foram mensurados, em duplicata, e serviram de base para o cálculo do IMC. Para classificar o estado nutricional do adulto foi utilizado o Índice de Massa Corporal (IMC), categorizado em: peso adequado: IMC $\leq 24,9$, sobrepeso: IMC $25-29,9 \mathrm{Kg} / \mathrm{m}^{2}$ e obesidade: IMC $\geq 30 \mathrm{Kg} / \mathrm{m}^{2}$ (WHO, 2015).

Foram aferidos também a circunferência da cintura (CC), a qual foi classificada de acordo com o seguinte critério: normal: $<80$ mulheres e $<94$ homens, aumentada: $\geq 80<88$ mulheres e $\geq 94<102$ homens e muito aumentada: $\geq 88$ mulhers e $\geq 102$ homens; para obtenção da CC, utilizou-se como referência a menor curvatura entre o rebordo costal e a crista ilíaca, fazendo as medidas por fita inextensível em $\mathrm{cm}$ e classificando os resultados segundo a OMS (WHO, 2015).

Em relação as morbidades, essas foram classificadas de acordo com os parâmetros bioquímicos inadequados, a saber: triglicerídeos (mg/dL) - normal: $<150 \mathrm{mg} / \mathrm{dL}$ e alterado: $\geq 150 \mathrm{mg} / \mathrm{dL}$, colesterol Total (mg/dL) - normal: $<200 \mathrm{mg} / \mathrm{dL}$ e alterado: $\geq 200 \mathrm{mg} / \mathrm{dL}$, LDL-c $(\mathrm{mg} / \mathrm{dL})$ normal: $<160 \mathrm{mg} / \mathrm{dL}$ e alterado: $\geq 160$ $\mathrm{mg} / \mathrm{dL}$, HDL-c $(\mathrm{mg} / \mathrm{dL})$ - normal: $\geq 40$ homens s e $\geq 50$ mulheres e $<40$ homens e $<50$ mulheres, colesterol não HDL (mg/dL) - normal: $<160 \mathrm{mg} / \mathrm{dL}$ e alterado: $\geq 160 \mathrm{mg} / \mathrm{dL}$ e glicemia em jejum (md/dL) normal: $<100$ $\mathrm{mg} / \mathrm{dL}$ e alterado: $\geq 100 \mathrm{mg} / \mathrm{dL}$. Os exames bioquímicos foram realizados por meio de coleta de sangue na veia cubital em um tubo com EDTA-K3E Vacutainer, após jejum noturno de 12 horas e analisados pelo método enzimático colorimétrico (Analisador automático de bioquímica - BA 400 Biosystems $^{\mathrm{TM}}$ ). As concentrações séricas de lipídeos foram categorizadas conforme os critérios da $V$ Diretriz Brasileira Sobre Dislipidemias e Prevenção da Aterosclerose (XAVIER HT, et al., 2013). E a glicemia de jejum foi classificada de acordo com a SOCIEDADE BRASILEIRA DE DIABETES (SBD) (MILECH A, 2016).

Inicialmente, foram efetuadas análises descritivas, a fim de caracterizar a distribuição de frequência das variáveis em estudo. A categoria das variáveis que apresentou a melhor condição ou a menor prevalência da HAS foi considerada como referência para as análises. A associação dos possíveis fatores preditivos da HAS foi inicialmente avaliada por análises bivariadas. As variáveis que apresentarem nesta análise um valor de $p<0,20$ no teste de Poisson simples foram selecionadas para o modelo multivariado. As análises foram realizadas por meio de Regressão de Poisson, com ajuste robusto do erro padrão. Por serem consideradas variáveis colineares, o IMC e a CC $(r 2=0,75)$, optou-se por manter na regressão de Poisson ajustada apenas o IMC.

As análises seguiram um modelo teórico de determinação, hierarquizado em quatro blocos de variáveis: primeiro bloco, as variáveis socioeconômicas (escolaridade e ocupação), segundo bloco (variáveis biológicas e comportamentais - faixa etária e atividade física), terceiro bloco (variáveis antropometricas - IMC) e quarto bloco (variáveis de morbidades - triglicerídeos, colesterol total, colesterol não HDL e glicemia de jejum). Os resultados foram expressos por razões de prevalências (RP) ajustadas com os respectivos intervalos de confiança (IC 95\%), sendo considerado o nível de significância estatística de $5 \%(p<0,05)$. As análises estatísticas foram processadas com o auxílio de software Stata, versão 14.0.

Através do Comitê de Ética e Pesquisa em Seres Humanos do IMIP, a pesquisa maior foi aprovada sob protocolo no 3.201 - 12 e CAAE № 07246912.6.0000.5201, acordando com os preceitos da Resolução do Conselho Nacional de Saúde 466/12 (BRASIL, 2013). As informações tiveram caráter sigiloso, preservando a identidade das pessoas avaliadas na investigação, cuja adesão foi formalizada com o Termo de 
Consentimento Livre e Esclarecido (TCLE). Devido à escassez de estudos que tratem sobre comunidades urbanas que vivem em precárias condições de vida, bem como pelo crescente aumento de pessoas acometidas pelas DCNTs, faz-se necessário publicar esses dados, que mesmo com o lapso temporal, são atuais e podem fornecer bases para ações do poder público local, assim como outros estudos.

\section{RESULTADOS}

Esse estudo contou com a participação de 663 sujeitos, com uma média de idade de 40,6 (DP 10,7) anos para o sexo masculino e 40,3 (DP 11,0) anos para o feminino. A prevalência de hipertensão encontrada entre os adultos de 20 a 59 anos foi de $36,9 \%$.

Quando avaliado às variáveis socioeconômicas, constatou-se que as classes menos favorecidas financeiramente $(D / E)$ possuíam uma maior prevalência de HAS $(38,2 \%)$, enquanto que na mais rica $(B)$ somente $6 \%$ tinha hipertensão. Em relação as variáveis ambientais, especificamente as relativas ao saneamento básico (destino de lixo, destino de dejetos e abastecimento de água) para as categorias analisadas, todas as prevalências foram similares (cerca de 36\%) entre os sujeitos hipertensos (Tabela 1).

No que se refere às variáveis biológicas, verificou-se que houve uma predominância da doença entre os homens $(38,3 \%)$, quando comparado às mulheres $(36,2 \%)$, contudo essa diferença não foi significativa $(p=0,611)$. Avaliando os grupos etários, percebe-se um aumento progressivo das prevalências de HAS à medida que idade aumentava, com esse percentual chegando a $62 \%$ entre aqueles maiores de 50 anos. Para a variável cor, a maior frequência do desfecho foi para à categoria "preto" (39,5\%).

Em relação as variáveis comportamentais, quanto ao tabagismo constatou-se uma prevalência de hipertensão de $26,5 \%$ entre os ex-fumantes e fumantes. No que se refere ao consumo de sal, os resultados foram similares entre aqueles que tinham um consumo adequado ou inadequado, com prevalência de $36 \%$.

Tabela 1 - Distribuição das variáveis socioeconômicas, biológicas e comportamental da amostra, prevalência de hipertensão e respectivas razões de prevalência simples e IC 95\%. 2014.

\begin{tabular}{|c|c|c|c|c|c|c|c|}
\hline \multirow{2}{*}{ Variáveis } & \multicolumn{2}{|c|}{ Total } & \multicolumn{2}{|c|}{ HAS } & \multirow[b]{2}{*}{$\mathbf{R P}$} & \multirow{3}{*}{ IC 95\% } & \multirow{2}{*}{$\begin{array}{l}\text { Valor de } \\
p^{*}\end{array}$} \\
\hline & $\mathrm{N}(663)$ & $\%$ & n & $\%$ & & & \\
\hline & \multicolumn{6}{|c|}{ Socioeconômicas } & \\
\hline Escolaridade & & & & & & & 0,143 \\
\hline Analfabeto/fundamental 1 incompleto & 190 & 28,7 & 77 & 40,5 & 1,23 & $0,95-1,59$ & \\
\hline $\begin{array}{c}\text { Fundamental } 1 \text { completo/fundamental } 2 \\
\text { incompleto }\end{array}$ & 147 & 22,2 & 49 & 33,3 & 1,01 & $0,75-1,36$ & \\
\hline Fundamental 2 completo/médio incompleto & 113 & 17,0 & 49 & 43,4 & 1,31 & $0,99-1,75$ & \\
\hline $\begin{array}{l}\text { Médio completo/superior incompleto/superior } \\
\text { completo }\end{array}$ & 213 & 32,1 & 70 & 32,9 & 1,0 & - & \\
\hline Ocupação & & & & & & & 0,003 \\
\hline Trabalha & 431 & 65,0 & 148 & 34,3 & 1,0 & & \\
\hline Não Trabalha & 200 & 30,2 & 78 & 39,0 & 1,13 & $0,91-1,41$ & \\
\hline $\begin{array}{l}\text { Aposentado/Pensionista/ } \\
\text { Beneficiário }\end{array}$ & 32 & 4,8 & 19 & 59,4 & 1,72 & $1,26-2,37$ & \\
\hline & \multicolumn{7}{|c|}{ Biológicas } \\
\hline Faixa etária $(n=663)$ & & & & & & & \\
\hline $20-29$ anos & 134 & 20,2 & 17 & 12,7 & 1,0 & - & $<0,001$ \\
\hline 30-39 anos & 177 & 26,7 & 47 & 26,5 & 2,09 & $1,25-3,47$ & \\
\hline $40-49$ anos & 180 & 27,2 & 74 & 41,1 & 3,24 & $2,00-5,22$ & \\
\hline $50-59$ anos & 172 & 25,9 & 107 & 62,2 & 4,90 & $3,09-7,76$ & \\
\hline & \multicolumn{7}{|c|}{ Comportamental } \\
\hline Atividade física $(n=663)$ & & & & & & & 0,080 \\
\hline Ativo & 522 & 78,7 & 184 & 35,2 & 1,0 & - & \\
\hline Insuficientemente ativo & 141 & 21,3 & 61 & 43,3 & 1,22 & $0,91-1,63$ & \\
\hline
\end{tabular}

Notas: * Teste de chi-quadrado de Pearson; Foram analisadas também as seguintes variáveis, contudo seus dados não foram apresentados na tabela pois o valor de $p$ não atendia ao critério de $p<0,20$ : classe, abastecimento de água, destino do lixo, esgotamento sanitário, sexo, cor, consumo de sal e tabagismo. HAS - pressão arterial (PA) elevada quando PA sistólica $\geq 140$ e/ou PA diastólica $\geq 90 \mathrm{mmHg}$ e uso de medicação; Fonte: Ferreira LCCN, et al., 2021. 
Em relação às variáveis antropométricas e bioquímicas, entre os considerados hipertensos, para todas as variáveis analisadas, quase metade da amostra ou mais do que metade apresentaram alterações quanto aos parâmetros normais das variáveis (Tabela 2).

Tabela 2 - Distribuição das variáveis antropométrica e bioquímicas (morbidade) da amostra, prevalência de hipertensão e respectivas razões de prevalência simples e IC 95\%. 2014.

\begin{tabular}{|c|c|c|c|c|c|c|c|}
\hline \multirow[b]{2}{*}{ Variáveis } & \multicolumn{2}{|c|}{ Total } & \multicolumn{2}{|c|}{ HAS } & \multirow[b]{2}{*}{$\mathbf{R P}$} & \multirow[b]{2}{*}{ IC $95 \%$} & \multirow{2}{*}{ Valor de $\mathbf{p}^{*}$} \\
\hline & $\mathbf{n}$ & $\%$ & $\mathbf{n}$ & $\%$ & & & \\
\hline & \multicolumn{7}{|c|}{ Antropométrica } \\
\hline $\operatorname{IMC}\left(\mathrm{Kg} / \mathrm{m}^{2}\right)(\mathrm{n}=660)$ & & & & & & & $<0,001$ \\
\hline$\leq 24,9$ & 198 & 30,0 & 42 & 21,2 & 1,0 & - & \\
\hline $25,0-29,9$ & 238 & 33,1 & 86 & 36,1 & 1,70 & $1,24-2,33$ & \\
\hline$\geq 30,0$ & 224 & 36,9 & 115 & 51,3 & 2,42 & $1,79-3,25$ & \\
\hline & \multicolumn{7}{|c|}{ Bioquímicas } \\
\hline Circunferência da Cintura (cm) $(\mathrm{n}=654)$ & & & & & & & $<0,001$ \\
\hline$<80(\mathrm{~F})$ e $<94(\mathrm{M})$ & 209 & 31,9 & 41 & 19,6 & 1,0 & - & \\
\hline$\geq 80<88(\mathrm{~F})$ e $\geq 94<102(\mathrm{M})$ & 111 & 17,0 & 43 & 38,7 & 1,97 & $1,37-2,83$ & \\
\hline$\geq 88(F)$ e $\geq 102(M)$ & 334 & 51,1 & 158 & 47,3 & 2,41 & $1,79-3,24$ & \\
\hline Triglicerídeos $(\mathrm{mg} / \mathrm{dL})(\mathrm{n}=515)$ & & & & & & & $<0,001$ \\
\hline$<150 \mathrm{mg} / \mathrm{dL}$ & 313 & 60,8 & 90 & 28,7 & 1,0 & - & \\
\hline$\geq 150 \mathrm{mg} / \mathrm{dL}$ & 202 & 39,2 & 112 & 55,4 & 1,92 & $1,55-2,38$ & \\
\hline Colesterol Total $(\mathrm{mg} / \mathrm{dL})(\mathrm{n}=517)$ & & & & & & & 0,033 \\
\hline$<200 \mathrm{mg} / \mathrm{dL}$ & 271 & 52,4 & 94 & 34,7 & 1,0 & - & \\
\hline$\geq 200 \mathrm{mg} / \mathrm{dL}$ & 246 & 47,6 & 108 & 43,9 & 1,26 & $1,01-1,57$ & \\
\hline Colesterol Não HDL (mg/dL) $(n=517)$ & & & & & & & 0,004 \\
\hline$<160 \mathrm{mg} / \mathrm{dL}$ & 314 & 60,7 & 107 & 34,0 & 1,0 & - & \\
\hline$\geq 160 \mathrm{mg} / \mathrm{dL}$ & 203 & 39,3 & 95 & 46,8 & 1,37 & $1,11-1,69$ & \\
\hline Glicemia em jejum (md/dL) $(n=517)$ & & & & & & & $<0,001$ \\
\hline$<100 \mathrm{mg} / \mathrm{dL}$ & 443 & 85,7 & 155 & 35,0 & 1,0 & - & \\
\hline$\geq 100 \mathrm{mg} / \mathrm{dL}$ & 74 & 14,3 & 47 & 63,5 & 1,81 & $1,46-2,24$ & \\
\hline
\end{tabular}

Notas: * Teste de chi-quadrado de Pearson; Foram analisadas também as seguintes variáveis, contudo seus dados não foram apresentados na tabela pois o valor de $p$ não atendia ao critério de $p<0,20$ : LDL-c e HDL-c. HAS - pressão arterial (PA) elevada quando PA sistólica $\geq 140$ e/ou PA diastólica $\geq 90 \mathrm{mmHg}$ e uso de medicação. Fonte: Ferreira LCCN, et al., 2021.

A Tabela 3 apresenta os fatores associados à HAS, após ajustes pelo modelo hierarquizado de análise da regressão de Poisson. Permaneceram associados à pressão arterial elevada: faixa etária, IMC e triglicerídeos, os quais se associaram estatisticamente ao desfecho em estudo $(p<0,05)$.

Tabela 3 - Razão de prevalência (RP) ajustada hierarquicamente, estimadas por regressão de Poisson, para prevalência de hipertensão arterial em adultos. 2014.

\begin{tabular}{|c|c|c|c|c|}
\hline Nívela $^{a}$ & Variáveis & RP ajustada & IC 95\% & Valor de $p$ \\
\hline \multicolumn{5}{|c|}{ Fatores Biológicos } \\
\hline 2 & Faixa etária (anos) & & & $<0,001$ \\
\hline & $20-29$ & 1,0 & 1,0 & \\
\hline & $30-39$ & 1,41 & $0,84-2,36$ & \\
\hline & $40-49$ & 2,14 & $1,32-3,47$ & \\
\hline & $50-59$ & 3,18 & $2,01-5,05$ & \\
\hline \multicolumn{5}{|c|}{ Fatores antropométricos } \\
\hline 3 & $\operatorname{IMC}\left(\mathrm{Kg} / \mathrm{m}^{2}\right)^{\mathrm{b}}$ & & & $<0,001$ \\
\hline & $\leq 24,9$ & 1,0 & 1,0 & \\
\hline & $25,0-29,9$ & 1,46 & $1,05-2,03$ & \\
\hline & $\geq 30,0$ & 1,89 & $1,38-2,60$ & \\
\hline \multicolumn{5}{|c|}{ Fatores de morbidade } \\
\hline 4 & Triglicerídeos (mg/dL) & & & $<0,001$ \\
\hline & $\begin{array}{l}<150 \mathrm{mg} / \mathrm{dL} \\
\geq 150 \mathrm{mg} / \mathrm{dL}\end{array}$ & $\begin{array}{c}1,0 \\
1,55\end{array}$ & $\begin{array}{c}1,0 \\
1,24-1,93\end{array}$ & \\
\hline
\end{tabular}

Notas: a: Nível 1 foi composto por escolaridade e ocupação, no entanto não permaneceram no modelo pois não foram significativas. Nível 2 foi ajustado por atividade física. Nível 3 foi ajustado por faixa etária do modelo 2. Nível 4 foi ajustado por faixa etária do nível 2 e IMC do nível 3; b: IMC - índice de massa corporal.

Fonte: Ferreira LCCN, et al., 2021. 


\section{DISCUSSÃO}

Nesse estudo, encontrou-se uma elevada prevalência de HAS de 36,9\%, em consonância com uma pesquisa realizada também em comunidade de vulnerabilidade, em adultos quilombolas na Bahia, que encontrou resultado semelhante, $38,5 \%$ e em estudo realizado em populações urbanas de baixa renda nos países de Gâmbia e Serra Leoa, localizados na África subsaariana, que também verificou elevada frequência do problema 44,8\% (SILVA TSS, et al., 2016; AWAD M, et al., 2014). A alta prevalência de HAS encontrada nos estudos, provavelmente está relacionada à baixa condição socioeconômica, que por sua vez está ligada a diversos fatores que contribuem para essa prevalência como: uma baixa taxa de tratamento da hipertensão; baixo nível de educação e conscientização; alta ingestão de sódio e baixa ingestão de potássio; bem como um aumento no nível de estresse.

Outro estudo realizado na Itália encontrou uma prevalência inferior $25,9 \%$, corroborando com outros estudos realizados no Brasil, que observaram frequências inferiores de HAS em São Paulo $(14,0 \%)$ e na Amazônia Legal (24,1\%) (TOCCI G, et al.; 2017; SILVA EC, et al., 2016; ZANGIROLANI LTO, et al., 2018). De acordo com dados do VIGITEL, essa prevalência encontrada, está acima do valor estimado para a população Brasileira (24,3\%) (BRASIL, 2017). Esse resultado evidencia a falta de reconhecimento da HAS como importante agravo de saúde pública e também mostra que a HAS ainda é um grande desafio a ser enfrentado.

Na comunidade dos Coelhos, não foram encontradas diferenças significativas na prevalência da HAS entre os sexos, diferentemente do que apontam alguns estudos em comunidades urbanas subnormais que ora apontam maior prevalência de HAS no sexo feminino, ora no sexo masculino (DANIEL OJ, et al., 2013; KHANAM MA, et al., 2015; HELELO TP, et al., 2014). No sexo feminino, essa maior prevalência poderia estar relacionada a fatores hormonais, como também ao uso de contraceptivos (PIMENTA E, 2012). Além do mais, dois terços da amostra de adultos na comunidade dos Coelhos foi composto por mulheres.

Outros reconhecidos fatores de risco para a HAS como atividade física, tabagismo e consumo de sal não estiveram associados no presente estudo. Tal fato pode estar relacionado à causalidade reversa, no qual a exposição estudada pode ser influenciada pela ocorrência do desfecho. Com relação ao consumo de sal, dados da VIGITEL encontrou prevalência semelhante de consumo inadequado (15,6\%), ou seja, pessoas que referiram ter consumo alto ou muito alto de sal, no entanto esse valor provavelmente está subestimado em ambas as pesquisas por ser uma medida auto-referida (BRASIL, 2014). Esse resultado per si vai de encontro ao relatado em outras pesquisas que mostram a alta ingestão de sódio da população brasileira (ARAUJO MC, et al., 2013; SARNO F, et al., 2013).

Quanto à faixa etária, pode-se perceber que em todos os grupos analisados a prevalência da HAS foi estatisticamente significativa, e que quanto maior a idade apresentada pelo grupo, maior foi a RP e a proporção de indivíduos que apresentava hipertensão, corroborando com outros estudos (ZANGIROLANI LTO, et al., 2018; HELELO TP, et al., 2014; MATAR D, et al., 2015). No grupo com faixa etária mais alta (5059 anos), mais de $60 \%$ dos participantes apresentavam a HAS, evidenciando a maior carga da doença nessa população apesar de ainda não serem idosos. Tal achado pode estar relacionado com as alterações hemodinâmicas da doença, como o aumento do débito ou da resistência periférica que inicia entre os 20 e 30 anos de idade, porém entre os 30 e 50 anos é que os níveis elevados se instalam (MALACHIAS MVB, et al., 2016).

Foi observado no presente estudo associação entre o excesso de peso e a HAS, resultado também encontrado em outras pesquisas nacionais e internacionais (ZANGIROLANI LTO, et al., 2018; BEZERRA VM, et al., 2017; PAPATHANASIOU G, et al., 2015). No Brasil, entre 2006 e 2017, a prevalência de sobrepeso aumentou de $42,6 \%$ para 54,0\% (incremento anual de 1,14\%) e a de obesidade passou de $11,8 \%$ para 18,9\% (incremento de $0,67 \%$ ao ano) (BRASIL, 2017). Nos Coelhos, $70 \%$ dos participantes foram identificados com excesso de peso (sobrepeso/obesidade). Dentre os participantes obesos, mais da metade (51,3\%) apresentava HAS.

Um estudo longitudinal, realizado nos Estados Unidos, verificou que entre os participantes que se tornaram obesos, a razão de prevalência encontrada para a associação com a elevação da pressão arterial foi de 4,17, e para quem tinha sobrepeso foi de 1,58, tais resultados ressaltam a importância do excesso de peso em 
aumentar o risco de hipertensão (SHIHAB HM, et al., 2012). Por ser uma condição modificável, faz-se ainda mais urgente a criação de estratégias efetivas para o enfrentamento dessa doença, que além de influenciar o aumento da prevalência de hipertensão, é uma condição que eleva o risco para várias patologias como câncer, diabetes mellitus tipo 2, doença renal crônica e desordens musculoesqueléticas (AFSHIN A, et al., 2017).

Em relação às variáveis bioquímicas, apenas os níveis elevados de triglicerídeos permaneceram diretamente associados à HAS, isso é atribuído por alguns estudos à associação dos TG's elevados ao maior risco de doenças cardiovasculares e mortalidade (NORDESTGAARD BG e VARBO A, 2014). De acordo com alguns trabalhos, níveis aumentados da relação TG/HDL-c estão relacionados à resistência à insulina e ao maior risco cardiometabólico (FLOWERS E, et al., 2015). No entanto no presente trabalho essa relação não foi avaliada.

A principal limitação desse trabalho é o seu delineamento transversal, que não permite estabelecer uma relação de causa e consequência entre as variáveis independentes e à variável desfecho (HAS). Outra limitação que merece destaque é o maior percentual de mulheres entrevistadas, o que sugere um viés de seleção. Uma provável explicação para esse fato está ligada a maior dificuldade em encontrar homens nos domicílios em comparação às mulheres.

É oportuno ressaltar, que no país, estudos que estimam a prevalência da HAS em aglomerados urbanos subnormais são ainda escassos, justificando assim a comparação do presente trabalho com estudos de prevalência da população de adultos em geral e populações subnormais do mundo. Dessa forma, sugere-se que mais trabalhos sejam realizados nessa população, a fim de elucidar se, e se sim, por que essa população difere da população residente em áreas urbanas mais favorecidas.

\section{CONCLUSÃO}

A prevalência da HAS nesse estudo foi maior (36,9\%) do que a apresentada na maioria dos estudos nacionais, cujas médias oscilam em torno de $20 \%$. Essa alta prevalência, associada aos fatores associados encontrados, impõe a necessidade de incluir este problema como prioridade de saúde pública nas comunidades carentes, tendo em vista a escassez de trabalhos nessa população, visando o enfrentamento do agravo com eficácia na população afetada pela HAS, reduzindo assim a sua incidência e o impacto das DCNTs.

\section{FINANCIAMENTO}

O presente estudo teve suporte financeiro do Ministério de Ciência e tecnologia/Fundação de Amparo à Ciência e Tecnologia do Estado de Pernambuco- FACEPE (APQ-2136-4.06/12) para financiamento da pesquisa e pela Coordenação de Aperfeiçoamento de Pessoal de Nível Superior - CAPES através da concessão da bolsa de estudos.

\section{REFERÊNCIAS}

1. ALMEIDA ND. A saúde no Brasil, impasses e desafios enfrentados pelo Sistema Único de Saúde - SUS. Revista Psicologia e Saúde, 2013; 5(1):1-9.

2. ARAÚJO ASS, et al. Prevalência de hipertensão arterial autorreferida na população brasileira: análise da Pesquisa Nacional de Saúde, 2013. Revista Epidemiologia e Serviços de Saúde, 2015; 24(2):297-304.

3. ARAUJO MC, et al. Consumo de macronutrientes e ingestão inadequada de micronutrientes em adultos. Rev Saúde Pública, 2013; 47:177-89.

4. AFSHIN A, et al. Health Effects of Overweight and Obesity in 195 Countries over 25 Years. The New England Journal of Medicine, 2017; 377(1):13-27.

5. ASSOCIAÇÃO BRASILEIRA DE EMPRESAS DE PESQUISA (ABEP). Critério de classificação econômica Brasil. São Paulo: ABEP; 2014.

6. AWAD M, et al. Prevalence of hypertension in the Gambia and Sierra Leone, western Africa: a cross-sectional study. Cardiovascular Journal of Africa, 2014; 25(6): 269-278.

7. BEZERRA VM, et al. Comunidades quilombolas de Vitória da Conquista, Bahia, Brasil: hipertensão arterial e fatores associados. Cadernos de Saúde Pública, 2013; 29(9):1889-1902. 
8. BEZERRA VM, et al. Pré-hipertensão arterial em comunidades quilombolas do sudoeste da Bahia, Brasil. Cadernos de Saúde Pública, 2017; 33(10): e00139516.

9. BRASIL. Ministério da Saúde. Saúde Brasil 2010: uma análise da situação de saúde e de evidências selecionadas de impacto de ações de vigilância em saúde - Brasília, 2011. 372 p.: il. - (Série G. Estatística e Informação em Saúde). ISBN 978-85-334-1851-6.

10. BRASIL. Ministério da Saúde (MS). Conselho Nacional de Saúde. Resolução № 466, de 12 de dezembro de 2012. Diário Oficial da União, 2013; 13 dez.

11. BRASIL. Ministério da Saúde. Secretaria de Vigilância em Saúde. VIGITEL Brasil 2013: Vigilância de Fatores de Risco e Proteção para Doenças Crônicas por Inquérito Telefônico. Brasília: Ministério da Saúde, 2014.120 p.

12. BRASIL. Ministério da Saúde (MS). Vigitel Brasil 2017: Vigilância de fatores de risco e proteção para doenças crônicas por inquérito telefônico. Brasília: MS; 2018.

13. CHOR D, et al. Prevalence, awareness, treatment and influence of socioeconomic variables on control of high blood pressure: results of the ELSA-Brasil Study. PLoS One 2015; 10(6): e0127382.

14. CRAIG CL, et al. International physical activity questionnaire: 12 -country reliability and validity. Medicine \& Science in Sports \& Exercise, 2003; 35(8): 1381-1395.

15. DANIEL OJ, et al. Prevalence of Hypertension among Urban Slum Dwellers in Lagos, Nigeria. Journal of Urban Health, 2013; 90(6):1016-1025.

16. FLOWERS E, et al. Use of plasma triglyceride/high-density lipoprotein cholesterol ratio to identify increased cardiometabolic risk in young, healthy South Asians. Indian Journal Of Medical Research, 2015 Jan.; 141(1): 68-74.

17. HELELO TP, et al. Prevalence and Associated Factors of Hypertension among Adults in Durame Town, Southern Ethiopia. PLoS ONE, 2014; 9(11): 1-9.

18. KHANAM MA, et al. Prevalence and determinants of pre-hypertension and hypertension among the adults in rural Bangladesh: findings from a community-based study. BMC Public Health, 2015; 15(203).

19. MALACHIAS MVB, et al. 7º Diretriz Brasileiras de Hipertensão. Arquivos Brasileiro de Cardiologia, 2016; 107(Supl. 3): 1-83.

20. MALTA DC, et al. Prevalência e fatores associados com hipertensão arterial autorreferida em adultos brasileiros. Revista de Saúde Pública, 2017; 51 (Supl1):11s.

21. MARINHO F, et al. Novo século, novos desafios: mudança no perfil da carga de doença no Brasil de 1990 a 2010. Revista Epidemiologia e Serviços de Saúde, 2016; 25(4):713-724.

22. MATAR D, et al. Prevalence, Awareness, Treatment, and Control of Hypertension in Lebanon. The Journal of Clinical Hypertension, 2015; 17(5): 381-388.

23. MILECH A, et al. Diretrizes da Sociedade Brasileira de Diabetes (2015-2016). São Paulo: 2016.

24. MOREIRA JPL, et al. A prevalência de hipertensão arterial sistêmica autorreferida nos ambientes urbano e rural do Brasil: um estudo de base populacional. Cadernos de Saúde Pública, 2013; 29(1): 62-72.

25. NORDESTGAARD BG, VARBO A. Triglycerides and cardiovascular disease. The Lancet, 2014; 384: 626-635.

26. OLSEN MH, et al. A call to action and a lifecourse strategy to address the global burden of raised blood pressure on current and future generations: the Lancet Commission on hypertension. The Lancet, 2016; 388(10060), 2665-2712.

27. PAPATHANASIOU G, et al. Association of high blood pressure with body mass index, smoking and physical activity in healthy young adults. The Open Cardiovascular Medicine Journal, 2015; 9: 5-17.

28. PIMENTA E. Hypertension in women. Hypertension Research, 2012; 35(2):148-52.

29. SÁ NNB, MOURA EC. Excesso de peso: determinantes sociodemográficos e comportamentais em adultos, Brasil, 2008. Cadernos de Saúde Pública, 2011; 27(7):1380-1392.

30. SARNO, F. et al. Estimativa de consumo de sódio pela população brasileira, 2008-2009. Revista de Saúde Pública, 2013; 47(3):571-578.

31. SILVA TSS, et al. Hipertensão arterial e fatores associados em uma comunidade quilombola da Bahia, Brasil. Cadernos de Saúde Coletiva, 2016; 24(3): 376-383.

32. SILVA EC, et al. Prevalência de hipertensão arterial sistêmica e fatores associados em homens e mulheres residentes em municípios da Amazônia Legal. Revista Brasileira de Epidemiologia, 2016; 19(1): 38-51.

33. SHIHAB HM, et al. Body Mass Index and Risk of Incident Hypertension Over the Life Course: The Johns Hopkins Precursors Study. Circulation, 2012; 126(25): 2983-2989.

34. TOCCI G, et al. Prevalence and control of hypertension in the general practice in Italy: updated analysis of a large database. Journal of Human Hypertension, 2017; 31 (4): 258-262.

35. UNIVERSIDADE FEDERAL DE PERNAMBUCO. Centro de Ciências da Saúde. Departamento de Nutrição. III Pesquisa estadual de saúde e nutrição: saúde, nutrição, alimentação, condições socioeconômicas e atenção à saúde no estado de Pernambuco, 2006. Recife: 2012.

36. VEENSTRA G. Race, gender, class, sexuality (RGCS) and hypertension. Social Science \& Medicine, 2013; 89: 16-24.

37. WORLD HEALTH ORGANIZATION (WHO). Health statistics and information systems: estimates for 2000-2012: cause specific mortality. Geneva: WHO; $2015 . \quad$ Disponível em: http://www.who.int/healthinfo/global_burden_disease/estimates/en/index1. html. Acesso em: 23 nov. 2020.

38. WORLD HEALTH ORGANIZATION (WHO). Obesity: Preventingand managing the global epidemic - Report of a WHO consultation on obesity. Geneva: WHO; 1998.

39. XAVIER HT, et al. V Diretriz Brasileira de Dislipidemias e Prevenção da Aterosclerose. Arquivos Brasileiro de Cardiologia. 2013; 101(4 Supl. 1): 1-20.

40. ZANGIROLANI LTO, et al. Hipertensão arterial autorreferida em adultos residentes em Campinas, São Paulo, Brasil: prevalência, fatores associados e práticas de controle em estudo de base populacional. Ciência \& Saúde Coletiva, 2018; 23(4): 1221-1232. 